\title{
Using Limiting Distribution of Transition Probability to Assess Coexisting Inflow and Outflow Stenosis in Experimental Stenotic Arteriovenous Grafts
}

\author{
${ }^{1}$ Wei-Ling Chen, ${ }^{2}$ Yi-Chen Mai, ${ }^{3}$ Chien-Ming Li, ${ }^{4}$ Chung-Dann Kan, ${ }^{5}$ Hung-Shang Liu, and ${ }^{6}$ Chia-Hung Lin \\ ${ }^{1}$ Department of Engineering and Maintenance, Kaohsiung Veterans General Hospital, Kaohsiung City, 81362, Taiwan. \\ E-mail: lynnchen.k@gmail.com. \\ ${ }^{2}$ Department of Aeronautics and Astronautics, National Cheng Kung University, Tainan City, 70101, Taiwan. \\ E-mail: cancersign810712@gmail.com. \\ ${ }^{3}$ Division of Infectious Diseases, Department of Medicine of Chi Mei Medical Center, Tainan City, 710, Taiwan. \\ E-mail: 235813cmli@gmail.com. \\ ${ }^{4}$ Department of Surgery, National Cheng Kung University Hospital, College of Medicine, National Cheng Kung University, \\ Tainan City, 70101, Taiwan. E-mail: kcd56@mail.ncku.edu.tw. \\ ${ }^{5}$ Department of Internal Medicine, Kaohsiung Veterans General Hospital, Tainan Branch, Tainan City, 71051, Taiwan. \\ E-mail: Liu04220422@yahoo.com.tw \\ ${ }^{6}$ Department of Electrical Engineering, Kao-Yuan University, Kaohsiung City, 82151, Taiwan. E-mail: eechl53@gmail.com.
}

\begin{abstract}
This study proposes a screening model to assess coexisting inflow and outflow stenosis in an experimental arteriovenous graft (AVG). Early clots and thrombosis lead to stenosis progression and increase variations in inflow and outflow pressures, flow velocities, and flow resistances. A substitution rate matrix (SRM) is used to replace the pressure variations and derive a transition probability matrix (TPM). The limitation values of transition probabilities can be used to identify the normal conditions and flow instabilities using the Euclidean distance estimation method. The joint probability could then be specified as a critical threshold to identify the stenosis levels in the assessment of coexisting stenosis.
\end{abstract}

Index Terms - Arteriovenous Graft, Substitution Rate Matrix, Transition Probability Matrix.

\section{Introduction}

Cardiovascular diseases are major risk factors for morbidity and mortality in patients with both end-stage renal disease and diabetes. Dialysis patients who suffer from progressive atherosclerosis can develop stenosis in the lumen of the vascular wall at the arterial site or venous site. Thus, they can develop venous and arterial stenosis or vascular diseases, which can include complications such as hypertension, hyperlipidemia, dyslipidemia, and diabetes mellitus. In addition, an arteriovenous fistula (AVF) or an arteriovenous graft (AVG) is vital for delivering an adequate flow rate of $>600 \mathrm{ml} / \mathrm{min}$. Due to repeat puncturing in the vascular access or long-term use, early clots, thrombosis, and progression of atherosclerosis result in reduced blood flow. These complications could lead to venous (outflow) stenosis, arterial (inflow) stenosis, and ultimately progression of coexisting stenosis at the inflow and outflow sites, as shown by their structures in Figure 1. In clinical examinations, AVGs have a higher infection rate and a higher patency rate than AVFs in stenosis growth at the venous anastomosis site, leading to venous neointimal hyperplasia and thrombosis [1-2]. Therefore, an acute problem will affect the cardiovascular system and the dialysis circuit, including hemodynamic variations in blood
Experimental Arteriovenous Graft

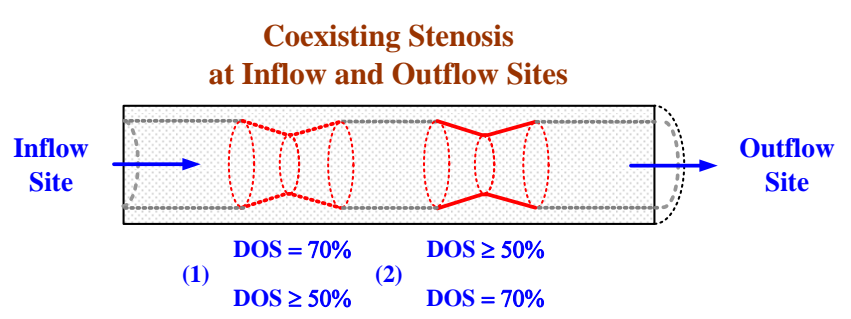

Figure 1. The structure of coexisting stenosis at inflow and outflow sites

flow, pressures, and flow velocities in the stenotic vicinity. Some studies [3-5] have proposed mathematical models or analytical solutions to analyze steady flow through a narrowed access or pulsatile flow in multiple stenotic accesses.

Numerical analyses have been performed to validate the correlation between in-vivo and in-vitro experiments. The Newtonian flow is through an elastic stenotic access, while the blood flow is characterized by the generalized power law model. Poiseuille's law [6-7] indicates a conversion from potential to kinetic energy. Thus, instability and turbulent flow in a stenotic route result in increases in pressure, pressure gradient, velocity, resistance, and wall stress. These increases can allow researchers to understand the important effects, impacts, and risks and then deal with real clinical problems. Dimensionless numbers, such as the Reynolds (Re) number, Womersley number, and Strouhal number [8-9], can also be used to characterize flow patterns, including steady laminar flow, transitional flow, and pulsatile flow. However, these analytical methods cannot be applied in the patient environment.

In real-time or online hemodynamic analysis, a narrowed access or higher degree of stenosis (DOS) limits the blood flow through the vascular access and increases access pressures in inflow sites, flow velocities, and flow resistances. Then, the pressure differences gradually increase between the inflow and outflow sites, while the hemodynamic condition has an outflow or coexisting stenosis at the inflow and outflow sites. The substitution rate matrix (SRM) method [10-13] was used for Euclidean 

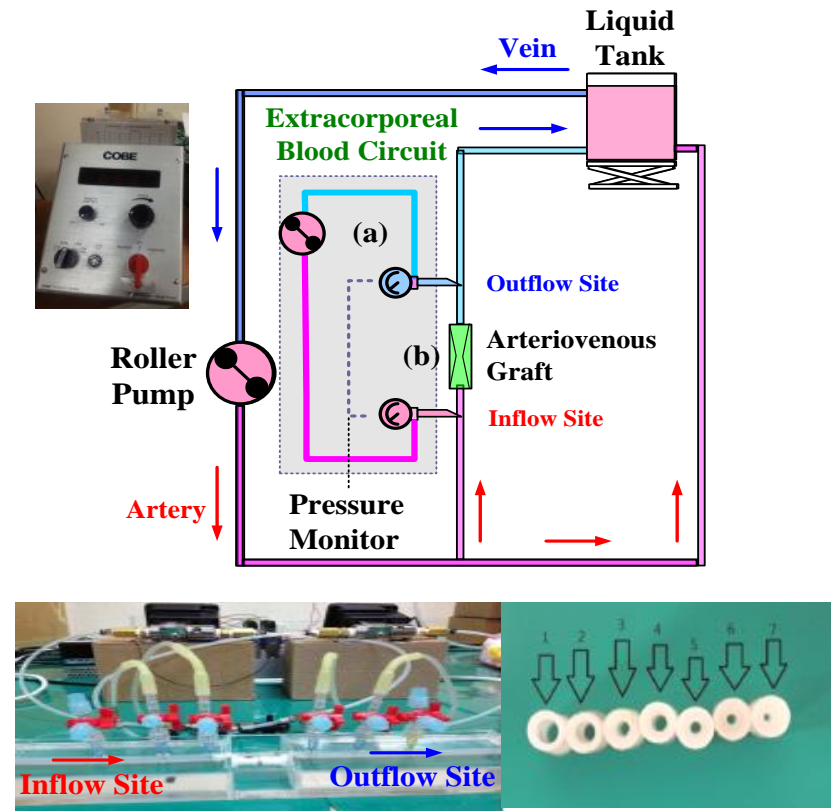

Figure 2. The circulation system in an experimental study

distance estimation and sequence analysis with pressure variations from the inflow to the outflow site. The Markov chain with two states of hemodynamic conditions expresses a matrix of substitution rates to replace the pressure variations. Then, the TPM can be derived. The SRM model screens the inflow and outflow pressure variations, while transition probabilities approach the same limitation value as the intra-hemodynamic stability over a finite timing interval. Different limitation values in the transition probabilities are used to separate normal conditions from flow instabilities under $\geq 50 \%$ stenosis using the distance estimation method. These quantities can be specified as gross variations to assess stenosis progression in an experimental stenotic graft, particularly in coexisting inflow and outflow stenosis.

\section{Experimental Setup}

Figure 2 shows an experimental circulation system model representing the mock flow phenomena of a HD patient's circulation system. The experimental system consists of a roller pump as a pressure source regulator (Precision blood pump, COBE, Lakewood, CO, USA), a liquid tank, silicone tubes, and a stenotic tube (50-95\% of tube diameter) (Figure 2). The roller pump (40-80 rpm) is used to drive the fluid mixture for an adult during HD condition with a flow rate of approximately $600 \mathrm{ml} / \mathrm{min}$, including the heart rate (80-120 beats/min), blood pressure, blood flow, and pulsatile flow. The temperature in our experimental study is about $28^{\circ} \mathrm{C}$ (steady state). The mixed water-glycerin fluid, with a hematocrit ratio of 38-62\%, kinematic viscosity of $3.2 \times 10^{-6} \mathrm{~m}^{2} / \mathrm{s}$, and density of $1090 \mathrm{~kg} / \mathrm{m}^{3}$ at $28^{\circ} \mathrm{C}$, was used as a blood-mimicking fluid (BMF), similar to a normal adult. According to the practical HD condition, a blood flow of $600 \mathrm{ml} / \mathrm{min}$ and a rotor speed of 40-60 rpm were used in this study.

The inflow and outflow pressures were measured using (a)

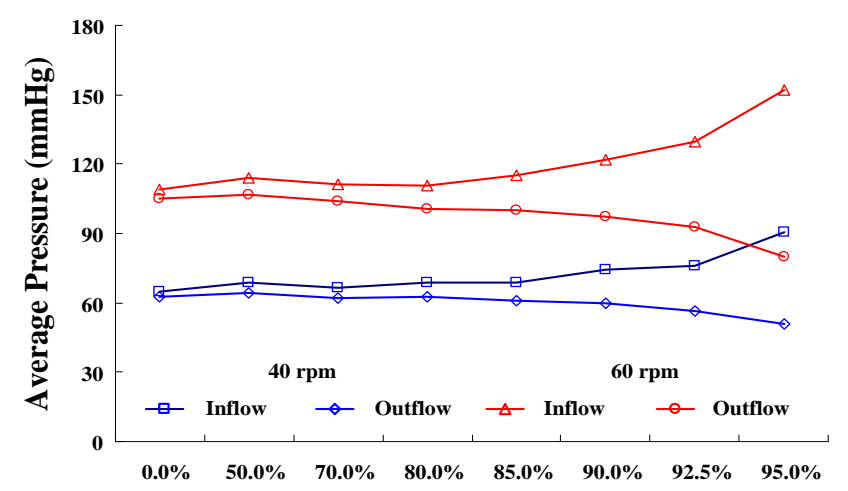

(b)

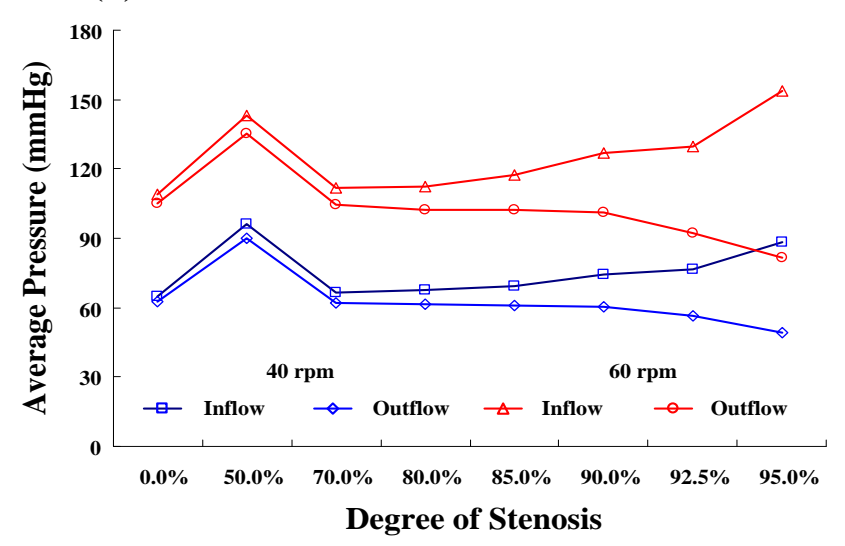

Figure 3. Inflow and outflow pressure measurements under 600 $\mathrm{ml} / \mathrm{min}$ and rotor speed, $40-60 \mathrm{rpm}$. (a) Inflow stenosis: $70 \%$ and Outflow stenosis: $0-95 \%$, (b) Inflow stenosis: $0-95 \%$ and Outflow stenosis: $70 \%$

venous needles, pressure transducers, and pressure sensors, as shown in Figure 2. Measurement data were transferred to a compatible PC or a tablet for further analysis using a data acquisition card (National Instruments Compact DAQ-9178 card, analog-to-digital converter, 8 channels, and $1 \mathrm{MHz}$ sampling rate). The inflow and outflow pressure signals, $P_{\text {mea }}$, were extracted using the detrending process [14-15], as follows:

$$
P_{\text {mea }}(t)=P_{\text {org }}(t)-\operatorname{det} \operatorname{rend}\left(P_{\text {org }}(t)\right)
$$

where syntax detrend $(\bullet)$ is a function of the detrending process used to remove unwanted and unpredictable variations such as the influence of the roller pump (harmonics) and transverse vibration pressures [16-17]; $P_{\text {mea }}(t)$ is the determined pressure from the incoming pressure signal; $P_{\text {org }}(t)$ is measured via the pressure sensors; and $t$ is the finite timing interval (sampling window length) represented by $t \in[0, T] ; T=10 \mathrm{~s}$ in this study.

The pressures $(\mathrm{mmHg})$ were measured at the inflow and outflow sites using pressure sensors that were continually monitored with pressure screening. We made 64 practical measurements at the inflow and outflow sites. For primary hemodynamic analysis, the inflow stenosis was $70 \%$ and the average inflow pressures steadily increased from the outflow stenosis from $50 \%$ to $95 \%$, while the outflow pressures gradually decreased. In addition, the outflow stenosis was $70 \%$, the inflow pressures steadily increased 


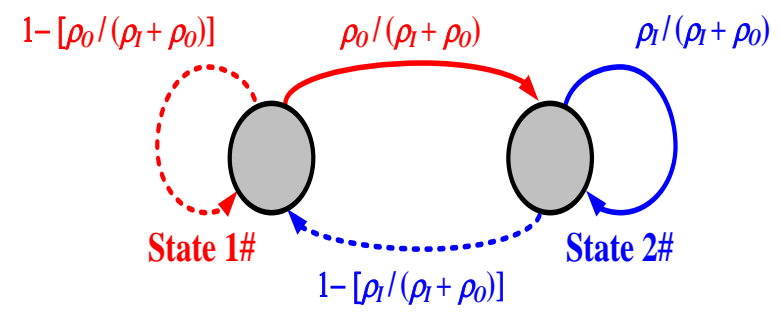

Figure 4. Markov chain with two states

from the inflow stenosis from $70 \%$ to $95 \%$, and the outflow pressure gradually decreased (Figure 3 ). It can be observed that the pressure differences, $\Delta P=\left|P_{I, \text { mea }}-P_{O \text {, mea }}\right|$, also gradually increased and reached about $40-72 \mathrm{mmHg}$, in terms of DOS from $0.0 \%$ to $95.0 \%$. The DOS is defined as follows [18]:

$$
\text { DOS \% }=\left[1-\left(\frac{d_{a}}{D_{a}}\right)^{2}\right] \times 100 \%
$$

where $D_{a}$ is the diameter of the normal access or graft in the direction of the BMF flow and $d_{a}$ is the diameter of the stenotic segment. Significant pressure variations were observed, while the inflow and outflow stenosis coexisted as the DOS increased. Therefore, we propose the substitution rate to derive the transition probabilistic model, and its limitation value can be used to identify stenosis progression with inflow and outflow pressure variations.

\section{Transition Probability Matrix}

The SRM-based mathematical model is a probabilistic method to describe variations between the inflow pressure, $P_{I}$, and outflow pressure, $P_{O}$, while an outflow stenosis or a coexisting inflow and outflow stenosis occurs. The vessel compliance is a pressure-volume relationship, which is the ratio of the difference of the systolic to diastolic amplitude of the diameter to the amplitude of the pressure. Thus, an increase in the pressure is accompanied by a decrease in vessel compliance. The inflow and outflow pressure variations can be defined as

$$
\rho_{I}=\frac{P_{I, \text { mea }}}{P_{I, \text { nor }}} \text { and } \rho_{O}=\frac{P_{O, \text { mea }}}{P_{O, \text { nor }}}
$$

where $\rho_{I}$ and $\rho_{O}$ are the inflow and outflow pressure ratios for pressure measurements; $P_{I \text {, mea }}$ and $P_{O \text {, mea }}$ are the pressures at the inflow (I) or outflow (O) site; and $P_{I, n o r}$ and $P_{O \text {, nor }}$ are the pressures under the DOS $\% \leq 50 \%$.

With two states, the substitution rates for each pair of pressure ratios, $\rho_{I}$ and $\rho_{O}$, are the parameters, $\lambda_{1}$ and $\lambda_{2}$. The SRM can be defined as

$$
Q=\left[\begin{array}{cc}
-\rho_{I} & \rho_{I} \\
\rho_{O} & -\rho_{O}
\end{array}\right]=U \Lambda U^{-1}, \quad-\rho_{i i}=\rho_{i j} \text { for } i \neq j
$$

The spectral decomposition of $Q$ is [10]

$$
\Rightarrow Q=U \Lambda U^{-1}=\left[\begin{array}{cc}
1 & -\rho_{I} \\
1 & \rho_{O}
\end{array}\right]\left[\begin{array}{cc}
0 & 0 \\
0 & -\rho_{I}-\rho_{O}
\end{array}\right]\left[\begin{array}{cc}
\frac{\rho_{O}}{\Delta} & \frac{\rho_{I}}{\Delta} \\
\frac{-1}{\Delta} & \frac{1}{\Delta}
\end{array}\right]
$$

where $\Delta=\left(\rho_{I}+\rho_{O}\right)$. The TPM, $P(t)$, is derived for Markov chain with two states, and the general formulation is

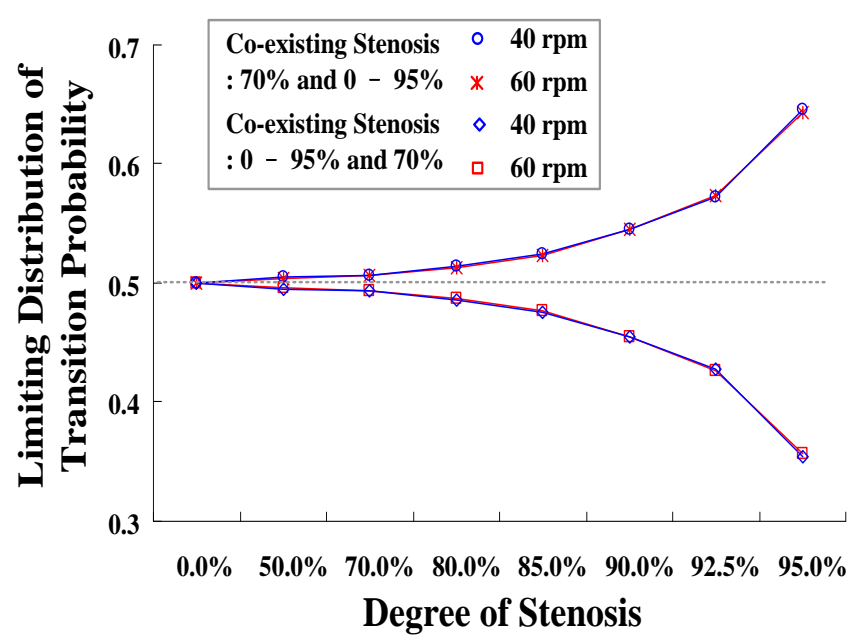

Figure 5. The limiting distribution of transition probability for various flow rates and DOSs

$$
\begin{gathered}
P(t)=e^{Q t}=\left[\begin{array}{cc}
1 & -\rho_{I} \\
1 & \rho_{O}
\end{array}\right]\left[\begin{array}{cc}
e^{0 \times t} & 0 \\
0 & e^{-\Delta \times t}
\end{array}\right]\left[\begin{array}{cc}
\frac{\rho_{O}}{\Delta} & \frac{\rho_{I}}{\Delta} \\
\frac{-1}{\Delta} & \frac{1}{\Delta}
\end{array}\right] \\
\Rightarrow P(t)=\frac{1}{\Delta}\left[\begin{array}{cc}
\rho_{O}+\rho_{I} e^{-\Delta t} & \rho_{I}-\rho_{I} e^{-\Delta t} \\
\rho_{O}-\rho_{O} e^{-\Delta t} & \rho_{I}+\rho_{O} e^{-\Delta t}
\end{array}\right] \\
\Rightarrow P(t)=\left[\begin{array}{ll}
p_{11}(t) & p_{12}(t) \\
p_{21}(t) & p_{22}(t)
\end{array}\right] \\
\text { subject to } 0 \leq p_{i j}(t) \leq 1, \sum_{j=1}^{2} p_{i j}(t)=1, \quad i=1,2
\end{gathered}
$$

As shown by the Markov chain in Figure 4, the limiting distribution is the stationary distribution, $\left[\rho_{O} / \Delta, \rho_{I} / \Delta\right]$, as

$$
\begin{aligned}
& \lim _{t \rightarrow \infty} p_{11}(t)=\lim _{t \rightarrow \infty} \frac{\rho_{O}}{\Delta}+\frac{\rho_{I}}{\Delta} e^{-\Delta t}=\frac{\rho_{O}}{\rho_{I}+\rho_{O}} \\
& \lim _{t \rightarrow \infty} p_{22}(t)=\lim _{t \rightarrow \infty} \frac{\rho_{I}}{\Delta}+\frac{\rho_{O}}{\Delta} e^{-\Delta t}=\frac{\rho_{I}}{\rho_{I}+\rho_{O}}
\end{aligned}
$$

For an ideal model, $\rho_{I}=\rho_{O}=1.0$, when time $t$ increases from 0 to $\infty$, the diagonal elements, $p_{11}(t)$ and $p_{22}(t)$, decrease from 1.0 to 0.5 , while the off-diagonal elements, $p_{12}(t)$ and $p_{21}(t)$, increase from 0.0 to 0.5 . Hence, the limiting distributions are $0.5,0.5,0.5$, and 0.5 . The mean value, $\mu=0.50$, can thus be obtained. Thus, the transition probabilities approach a value of 0.50 under the intra-hemodynamic stability (Figure 5). For coexisting stenosis, the pressure difference significantly increases and the limitation values of transition probabilities diverge from 0.50 for all inflow and outflow pressure ratios under different flow rates (40-60 rpm).

The distance estimation method is used to screen the similarity between the limitation value of transition probabilities, $p_{11}(t)$ and $p_{22}(t)$, as follows:

$$
\begin{aligned}
& d_{11}=\sqrt{\left(\lim _{t \rightarrow \infty} p_{11}(t)-\mu_{11}\right)^{2}}, \mu_{11}=0.50 \\
& d_{22}=\sqrt{\left(\lim _{t \rightarrow \infty} p_{22}(t)-\mu_{22}\right)^{2}}, \mu_{22}=0.50
\end{aligned}
$$

The joint probability, $p_{I O}$, accommodating the varying pressure ratios is given by the following formula: 
Table 1. Experimental results, comparisons with the proposed screening model and hemodynamic analysis (60rpm)

\begin{tabular}{|c|c|c|c|c|c|c|c|c|c|c|c|c|c|c|}
\hline \multicolumn{2}{|c|}{ DOS\% } & \multicolumn{2}{|c|}{ Pressure, $\mathrm{mmHg}$} & \multirow{2}{*}{$\begin{array}{c}\Delta P \\
\mathrm{mmHg}\end{array}$} & \multicolumn{2}{|c|}{ Pressure Ratio } & \multirow{2}{*}{$\begin{array}{c}V_{\text {est }} \\
\mathrm{m} / \mathrm{sec}\end{array}$} & \multirow{2}{*}{$\operatorname{Re}$} & \multirow{2}{*}{$\begin{array}{l}\text { Resistive } \\
\text { Index, Res }\end{array}$} & \multicolumn{2}{|c|}{ Eigenvalue } & \multicolumn{2}{|c|}{ Limitation } & \multirow[b]{2}{*}{$p_{I O}$} \\
\hline Inflow & Outflow & Inflow & Outflow & & $\rho_{I}$ & $\rho_{O}$ & & & & $\lambda_{1}$ & $\lambda_{2}$ & $p_{11}$ & $p_{22}$ & \\
\hline 0.0 & 0.0 & 109.25 & 104.85 & 4.40 & 1.00 & 1.00 & 1.048 & 724.76 & 0.333 & -2.00 & 0.00 & 0.50 & 0.50 & 1.0000 \\
\hline 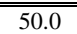 & \multirow{6}{*}{70.0} & 143.30 & 135.51 & 7.79 & 1.31 & 1.29 & 1.395 & 964.12 & 0.498 & -2.60 & 0.00 & 0.49 & 0.50 & 0.9946 \\
\hline 70.0 & & 111.88 & 104.56 & 7.32 & 1.02 & 0.99 & 1.353 & 934.89 & 0.483 & -2.01 & 0.00 & 0.49 & 0.51 & 0.9825 \\
\hline 80.0 & & 112.63 & 102.53 & 10.10 & 1.03 & 0.98 & 1.589 & 1097.99 & 0.559 & -2.01 & 0.00 & 0.48 & 0.51 & 0.9326 \\
\hline 85.0 & & 117.34 & 102.45 & 14.89 & 1.07 & 0.98 & 1.929 & 1333.42 & 0.637 & -1.98 & 0.00 & 0.48 & 0.52 & 0.8386 \\
\hline 90.0 & & 126.73 & 101.37 & 25.36 & 1.16 & 0.97 & 2.518 & 1740.09 & 0.722 & -2.13 & 0.00 & 0.45 & 0.55 & 0.4381 \\
\hline 92.5 & & 129.79 & 92.43 & 37.36 & 1.19 & 0.88 & 3.056 & 2112.04 & 0.771 & -2.07 & 0.00 & 0.43 & 0.57 & 0.1115 \\
\hline \multirow{7}{*}{70.0} & "50.0 & 113.84 & 106.90 & 6.94 & 1.04 & 1.02 & 1.317 & 910.23 & 0.468 & -2.06 & 0.00 & 0.49 & 0.50 & 0.9882 \\
\hline & 70.0 & 111.52 & 104.18 & 7.34 & 1.02 & 0.99 & 1.354 & 936.09 & 0.483 & -2.01 & 0.00 & 0.49 & 0.50 & 0.9819 \\
\hline & 80.0 & 110.95 & 100.86 & 10.09 & 1.01 & 0.96 & 1.588 & 1097.59 & 0.559 & -1.97 & 0.00 & 0.48 & 0.51 & 0.9291 \\
\hline & 85.0 & 115.05 & 100.29 & 14.76 & 1.05 & 0.96 & 1.921 & 1327.44 & 0.636 & -2.04 & 0.00 & 0.48 & 0.52 & 0.7939 \\
\hline & 90.0 & 121.67 & 97.35 & 24.32 & 1.11 & 0.93 & 2.466 & 1703.89 & 0.716 & -2.07 & 0.00 & 0.45 & 0.54 & 0.4394 \\
\hline & 92.5 & 129.68 & 92.88 & 36.80 & 1.19 & 0.88 & 3.033 & 2096.03 & 0.769 & -2.07 & 0.00 & 0.43 & 0.57 & 0.1211 \\
\hline & 95.0 & 152.15 & 80.07 & 72.08 & 1.39 & 0.76 & 4.245 & 2933.52 & 0.835 & -2.15 & 0.00 & 0.35 & 0.65 & 0.0002 \\
\hline
\end{tabular}

Note: (1) $V_{e s t}=\sqrt{\frac{\left(P_{I, \text { mea }}-P_{O, \text { mea }}\right)}{4}}=\sqrt{\frac{\Delta P}{4}}, \Delta P=P_{I, \text { mea }}-P_{O, \text { mea }}$ is the pressure drop, $(2) \operatorname{Re} s=\frac{V_{e s t}-V_{0}}{V_{e s t}}$, where $V_{e s t}$ is the estimated peak systolic velocity $(\mathrm{m} / \mathrm{sec})$, and $V_{0}=0.7 \mathrm{~m} / \mathrm{sec}$ is the peak systolic velocity under control conditions.

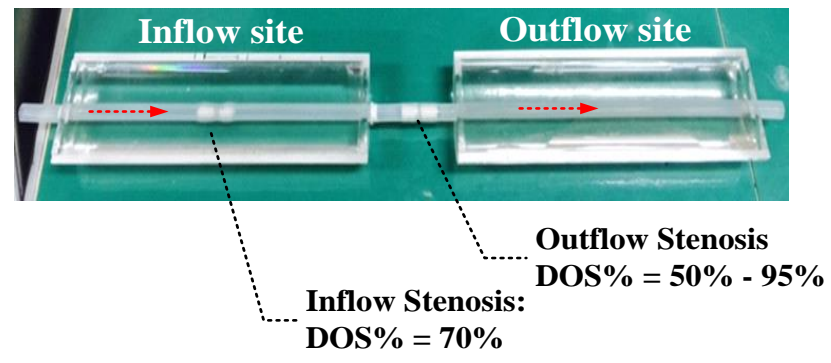

Figure 6. Stenotic segments in an experimental AVG

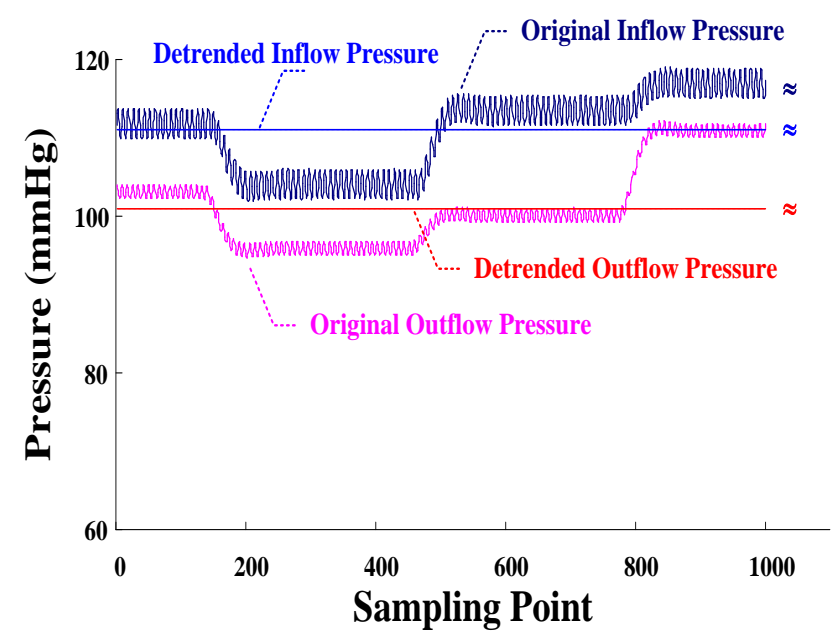

Figure 7. Original pressures and detrended pressures for coexisting stenosis $(\mathrm{DOS} \%=70 \%$ and $80 \%)$

$$
p_{I O}=\exp \left(-\frac{1}{2}\left(\frac{d_{11}}{\sigma}\right)^{2}\right) \times \exp \left(-\frac{1}{2}\left(\frac{d_{22}}{\sigma}\right)^{2}\right)
$$

where $\sigma=\mu \times 10 \%$ is the standard deviation. The joint probability, $p_{I O}>0.81$, was use to find the same states or similarity states for each pair of screening pressure ratios as the intra hemodynamic stability in an experimental graft. Otherwise, this indicates flow instability, while joint probability, $p_{I O}$, gradually approached zero. Thus, this index is calculated out to separate the normal condition (DOS\% < $50 \%$ ) from the coexisting inflow and outflow stenosis (DOS $\% \geq 50 \%$ ).

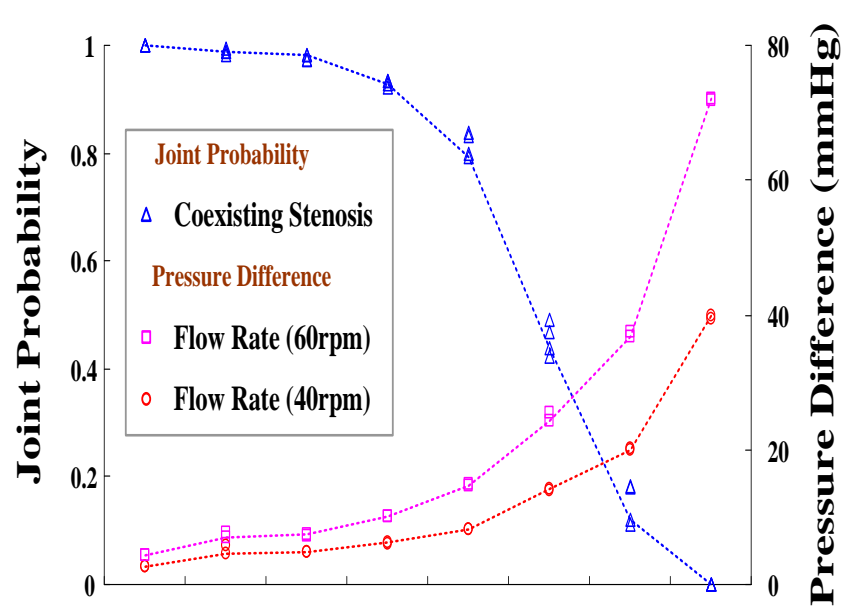

$\begin{array}{llllllll}0.0 \% & 50.0 \% & 70.0 \% & 80.0 \% & 85.0 \% & 90.0 \% & 92.5 \% & 95.0 \%\end{array}$

\section{Degree of Stenosis}

Figure 8 . Joint probability versus DOS and pressure difference versus DOS

\section{Simulation Results}

An experimental model mimicking the human cardiovascular circulation system was set up in the laboratory. A computer-assisted tool was designed to process inflow and outflow pressure signals, and the screening model was implemented using LabVIEW programming software (National Instruments ${ }^{\mathrm{TM}}$ Corporation, Austin, TX, USA) and Matlab software (Mathwork, Natick, MA, USA). A flow rate of $60 \mathrm{ml} / \mathrm{min}$ (roller pump, $40-60 \mathrm{rpm}$ ) and a heartbeat of 75 beats/min were used to drive the BMF through different stenotic segments, as seen in Figure 6. Pressure signals were obtained via a data acquisition card connected to a tablet or a PC. Original inflow and outflow pressures in the time domain were measured using pressure sensors, as shown by the sampling data in Figure 7 . Then, a detrending process was also used to remove unwanted influences and vibration pressures. For coexisting stenosis, the average inflow pressure was $110.95 \mathrm{mmHg}$ and the average outflow pressure was $100.86 \mathrm{mmHg}$, while the inflow and outflow narrowed degrees were $70 \%$ and $80 \%$, 
respectively, in the vascular access. For example, given the average pressures, $P_{I, \text { mea }}=110.95 \mathrm{mmHg}$ and $P_{O, \text { mea }}=100.86$ $\mathrm{mmHg}$, the screening procedure was as follows:

Step 1) compute the inflow and outflow pressure ratios, $\rho_{I}=1.01$ and $\rho_{O}=0.96$, using the equation (3),

Step 2) compute the substitution rates, $\lambda_{1}=-1.97$ and $\lambda_{2}=$ 0.00 , using the equation (4),

Step 3) computed the transition probabilities using the equations, (6) and (8), and then the limitation values of transition probabilities can be obtained using equations, (10) and (11),

Step 4) compute the Euclidean distances with the equations, (12) and (13), and joint probability using the equation (14), the $p_{I O}=0.9291$ can be obtained, as shown in Table 1.

The results indicate the average value, $p_{I O}=0.9291$, used to identify the stenosis level. When the gross variations in the average probability dropped from 1.00 to $<0.81$, coexisting stenosis progression was confirmed, and when the gross variations reached $<0.64$, they represented a higher degree under various flow rates (40-60 rpm), as shown in Figure 8. The critical threshold, <0.64, suggests that transluminal angioplasty or surgical revision should be performed to enlarge the focal site using a balloon or to further to dilate the stenotic lesion. The experimental results are shown in Table 1. In addition, we also performed hemodynamic analysis to compute pressure differences, $\Delta P$, between the inflow and outflow sites, injection velocities, $V_{\text {est }}$, across the vascular access, and the resistive (Res) indexes, as seen in Table 1. Pressure differences were caused by viscous losses in laminar flow, resulting in injection velocity increases through the stenotic segment and gradual increase in the average Re numbers; under a transition flow to pulsatile flow, the average Re number was greater than 1000 , with DOS $>70 \%$. Pressure differences increased as the induced flow rate decreased, causing a resistance in the flow from 0.333 to 0.835 . Comparing the proposed method with the hemodynamic analysis, the average joint probabilities showed inverse relationships (monotone decrease) with the increase in stenosis levels, providing a promising index for screening stenosis progression.

\section{Conclusion}

This experimental study has designed a measurement system and a screening model to assess stenosis progression in an in-vitro AVG. The pressure sensors were used to measure the inflow and outflow pressures. Pressure differences between the inflow and outflow sites were positively correlated with DOS from $0 \%$ to $95 \%$. The SRM model replaced the pressure ratios and was used to screen for inflow and outflow pressure variations, and then the limitation values of transition probabilities were used to separate the normal conditions from the intra-hemodynamic instability. The joint probability can identify the stenosis levels in the assessment of coexisting stenosis. The results also indicate that the prototype system could be integrated into a clinical hemodialysis machine, without any need for additional devices.

\section{Acknowledge}

This work is supported in part by the Ministry of Science and Technology, Taiwan, under contract number: MOST 104-2221-E -244-010 and MOST 105-2218-E-075B-001, duration: August 1, 2015 $\sim$ February 28, 2017, and is also supported in part by the research grant of Kaohsiung Veterans General Hospital, Tainan Branch, under contract number: VHYK 105-15, duration: January 1, 2016 December 31, 2016.

\section{Appendix}

The proposed screening model was designed as a graphic user interface (GUI) using the LabVIEW graphical programming (National Instruments ${ }^{\mathrm{TM}}$ Corporation, Austin, Texas, U.S.) and Matlab software (Mathwork, Natick, Massachusetts, U.S.), as shown in Figure 9.
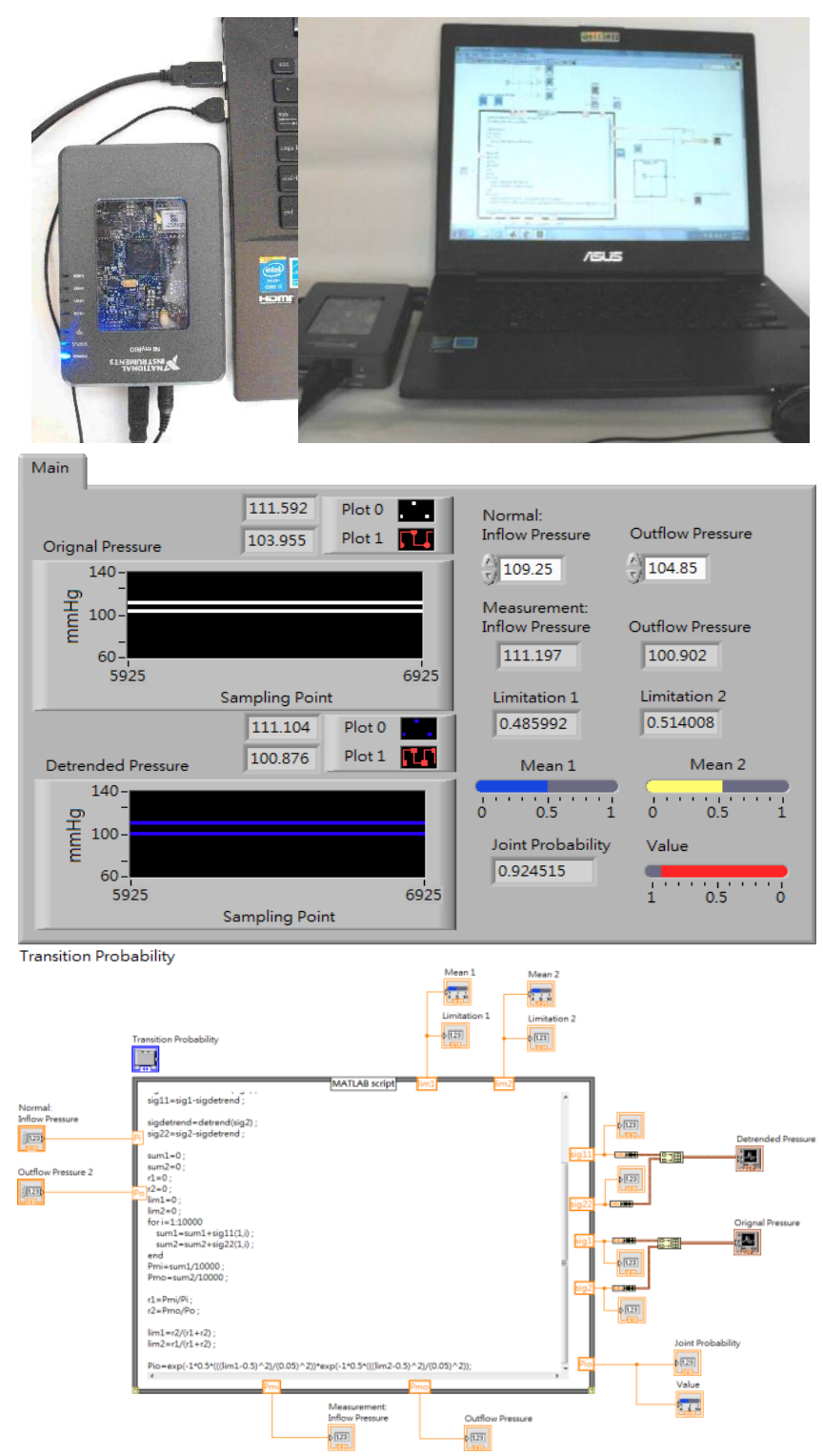

Figure 9. The proposed screening model was implemented in an embedded system and its graphic user interface (GUI) 


\section{Reference}

[1] I. Tricht, D. Wachter, J. Tordoir, and P. Verdonck, "Hemodynamics in a compliant hydraulic in vitro model of straight versus tapered PTFE aeterivenous graft, "Journal of Surgical research, vol. 116, no. 2, 2004, pp. 297-304.

[2] T. A. Manos, D. P. Sokolis, A. T. Giagini, C. H. Davos, J. D. Kakisis, E. P. Kritharis, N. Stergiopulos, and P. E. Karayannacos, S. Tsangaris, "Local hemodynamics and intimal hyperplasia at the venous side of a porcine arteriovenous shunt, "IEEE Trans Information Technology in Biomedicine, vol. 14, no. 3, 2010, pp. 681-90.

[3] Adarsh Kumar and Usha Awasthi, "A mathematical model for blood flow in a multiple stenosis artery, "Int. Journal of Math. Analysis, vol. 4, no. 50, 2010, pp. 2465-2472.

[4] Rekha Bali and Usha Awasthi, "A casson fluid model for multiple stenosed artery in the presence of magnetic field, "Applied Mathematics, vol. 2012, pp. 436-441.

[5] D. Biswas and U. S. Chakraborty, ",Pulsatile blood flow through a catheterized artery with an axially Nonsymmetrical stenosis," Applied Mathematical Sciences, vol. 4, no. 58, 2010, pp. 28652880.

[6] D. S. Sumner, Essential hemodynamic principles in Rutherford R. B. (ed): vascular Surgery, ed. 5, Philadelphia, Saunders, 2000, pp. 73-120.

[7] William D. Paulsona and Steven A. Jones, "Hemodynamics of the hemodialysis access: implications for clinical management," Contrib Nephrol. Basel, karger, vol. 142, 2004, pp. 238-253.

[8] E. L. Madsen, M. E. Deaner, and J. Mehi, "Properties of phantom tissue-like polymethyl-pentene in the frequency range $20-70 \mathrm{MHZ}$ , "Ultrasound Med Biol., vol. 37, no. 8, 2011, pp. 1327-1339.

[9] W. D. Paulsona and S. A. Jones, "Hemodynamics of the hemodialysis access: implications for clinical management, "Contrib Nephrol. Basel, karger, vol. 142, 2004, pp. 238-253.

[10] Ziheng Yang, Computational molecular evolution, Oxford Series in Ecology and Evolution, Oxford University Press, 2006.

[11] Matthew T. Jones, Estimating Markov transition matrices using proportions data: an application to credit risk, International Monetary Fund Working paper, November 2005, pp. 1-25.

[12] K. D. Makova, M. Ramsay, T. Jenkins, and W. H. Li, "Human DNA sequence variation in a $6.6-\mathrm{kb}$ region containing the melanocortin 1 receptor promotor, "Genetics, vol. 158, 2001, pp. 1253-1268.

[13] L. M. Mateiu and B. Rannala, "Inferring complex DNA substitution processes on phylogenies using uniformization and data augmentation, "Syst. Biol., vol. 55, 2006, pp. 259-269.

[14] Syntax: detrend, https://www.mathworks.com/help/matlab/ref/ detrend.html\#syntax.

[15] MATLAB® Data Analysis, MathWorks, Inc., https: //www. mathworks.com/help/pdf_doc/matlab/data_analysis.pdf.

[16] Mattias Holmer, Frida Sandberg, Kristian Solem, Egle Grigonyte, Bo Olde, and Leif Sornmo, "Extracting sardiac signal from the extracorporeal pressure sensors of a hemodialysis machine," IEEE Transactions of Biomedical Engineering, vol. 62, no. 5, May 2015, pp. 1305-1315.

[17] Hans-Dietrich Polaschegg, "Venous needle dislodgement:the pitfalls of venous pressure measurement and possible alternatives, a review, "Journal of Renal Care, vol. 36, no. 1, 2010, pp. 41-48.

[18] Wei-Ling Chen, Yu-Hsuan Lin, Chung-Dann Kan, Fan-Ming Yu, and Chia-Hung Lin, "Assessment of flow instabilities in in-vitro stenotic arteriovenous grafts using an equivalent astable multivibrator, "IET Science, Measurement \& Technology, vol. 9, no. 6, September 2015, pp. 709-716. 